\title{
Le développement du théâtre francophone au Québec à la fin du dix-neuvième siècle : le rôle de Louis-Honoré Fréchette (1839-1908)
}

Umut Incesu, Western University

En quatrième de couverture de La double vie littéraire de Louis Fréchette (2014) de JeanClaude Germain, on lit cette petite phrase : «Louis Fréchette! Ce nom fait partie de l'inconscient collectif des Québécois : on l'a donné à des rues, des écoles, des bibliothèques ». Fréchette est sans doute l'un des quelques noms qui sont parvenus jusqu'à nous de la littérature canadienne-française du dix-neuvième siècle. Bien qu'on le connaisse plutôt pour ses poèmes et ses contes, il a aussi été dramaturge. Il a écrit et adapté plusieurs textes pour la scène, qui ont connu une large diffusion et une réception positive au Canada. Ses pièces ont été publiées dans de grandes maisons d'édition de l'époque comme Beauchemin, et ont été représentées tant par des amateurs que par des troupes professionnelles dans de grandes salles de théâtre au Québec. On pense, entre autres, à Félix Poutré (1871), au Retour de l'exilé (1880), à Papineau (1880) et à Veronica (1974, publication posthume). Félix Poutré est la pièce la plus jouée par les amateurs au Canada français et chez les FrancoAméricains au dix-neuvième siècle (Germain 63). Cet article propose d'examiner le théâtre de Fréchette, plus précisément les modes de production de ses pièces (texte et représentation) et leurs réceptions. Il s'appuiera sur les critiques et des articles de journaux des années 1862 et 1880 afin d'étudier les représentations et de ressortir le nom des artistes et des techniciens du théâtre de l'époque, de même que les informations concernant la distribution des rôles, le jeu des acteurs, des actrices et le décor ${ }^{1}$. Il portera une attention particulière à l'interaction de Fréchette avec les gens de théâtre, notamment avec la célèbre actrice française Sarah Bernhardt, pour qui le dramaturge aurait écrit la pièce Véronica en collaboration avec l'auteur français Maurice de Pradel, aujourd'hui oublié (Germain 57).

\section{Le théâtre au Québec au dix-neuvième siècle}

Fréchette n'a pas été le seul écrivain à manifester son intérêt pour l'art de la scène au Québec du temps. De nombreux gens de lettres ont pris la plume pour écrire des textes dramatiques : Félix-Gabriel Marchand (1832-1900), ${ }^{2}$ Pamphile Le May (1837-1918), Régis Roy (1864-1944), Laure Conan (1845-1924) et Joséphine Marchand (1861-1925). Bien qu'à cette 
époque une écriture dramatique et une tradition théâtrale s'implantent, le théâtre d'expression française reste aujourd'hui peu connu, lacune qui a déjà été soulignée par les savants ${ }^{3}$. Depuis 2015, le projet de Bibliothèque et Archives nationales du Québec (BAnQ) « Théâtre à Montréal, 1825-1930» tente de remédier à cette situation en racontant l'histoire de quarante lieux de spectacles montréalais ${ }^{4}$. Des historiens et critiques s'intéressent au sujet, notamment Alex

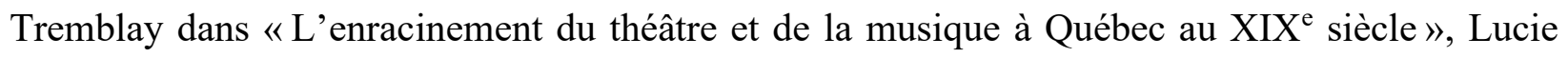
Robert, Jean-Marc Larrue, André G. Bourassa et André Duval.

L'ensemble de ces travaux permet de comprendre que le théâtre de langue française est en plein développement au Québec au dix-neuvième siècle grâce aux efforts des gens de théâtre, aux troupes de tournées et aux cercles dramatiques, pour ne citer qu'eux. On y remarque la naissance de nouvelles pratiques théâtrales et la création de plusieurs pièces à succès. Cependant, ce n'est qu'à partir de 1890 que l'art dramatique devient progressivement une pratique professionnelle (Larrue 25). L'établissement de salles de théâtres, le progrès du chemin de fer entre les États-Unis et le Canada favorisant la fréquentation des troupes de tournées, la croissance importante du public francophone sont autant de facteurs qui favorisent une expression plus large de ce théâtre. Dans cette perspective, les efforts des dramaturges pour produire des œuvres originales en français, notamment à partir de 1860 , et leur implication dans la vie théâtrale ont également été considérables. La production de Fréchette constitue un cas d'école.

\section{Louis-Honoré Fréchette et le théâtre}

Dramaturge, poète, journaliste, avocat et homme politique, Fréchette figure parmi les personnages les plus importants de l'histoire littéraire, culturelle et artistique au Québec pendant la seconde moitié du dix-neuvième siècle. Il fait ses études classiques entre 1854 et 1860 , et entreprend ensuite des études en droit à l'Université Laval, durant lesquelles il publie des vers et de la prose dans des périodiques et des revues comme Le journal de Québec et L'abeille (voir Bélanger). Ces poèmes sont rassemblés dans un recueil intitulé Mes loisirs, paru à Québec en 1863. Il ouvre son cabinet d'avocat à Lévis en 1864. Dans cette ville, il fonde les journaux Le drapeau de Lévis et Le journal de Lévis. Après la faillite de son cabinet, il s'exile à Chicago de 1866 à 1874, il poursuit ses activités de journalisme, collabore à L'observateur de Chicago et participe à la création de L'Amérique. Après son retour au Québec, Fréchette continue à pratiquer ses multiples métiers : il est d'abord élu député de Lévis pour le Parti libéral (1874-1878), relance ensuite sa 
carrière de journalisme dans La patrie, propriété d'Honoré Beaugrand, organe du Parti libéral. Fréchette y collabore avec ses poèmes et ses proses et en devient le rédacteur en chef en 1884. Quatre recueils de poésie sont publiés : La voix d'un exilé (1866), La découverte du Mississippi (1873), Pêle-mêle (1877) et Les fleurs boréales (1879). Ce dernier ouvrage vaut à Fréchette le prix Montyon de l'Académie française en 1880, prix littéraire prestigieux et accordé pour la première fois à un poète canadien qui fait de son auteur une célébrité dans le monde des lettres ${ }^{5}$. En plus des recueils de poèmes, Fréchette publie des contes, des nouvelles et des pamphlets, par exemple Originaux et détraqués (1892) et son recueil des contes Noël au Canada. Fréchette fréquente également le milieu littéraire tant en France qu'au Canada comme en témoigne sa correspondance ${ }^{6}$. Il est membre fondateur de la section française de la Société royale du Canada et président de l’École littéraire de Montréal. Répondant à une invitation de la ville de Rouen pour «participer aux célébrations du deuxième centenaire de la mort de Cavelier de La Salle », Fréchette se rend en France où il publie son ambitieux recueil La légende d'un peuple à Paris en 1887 (Germain 55). Il rencontre aussi des écrivains et des artistes, parmi lesquels Victor Hugo, le voyageur et traducteur Xavier Marmier, à qui Fréchette avait dédié son poème «La découverte du Mississippi », le dramaturge Émile Augier et le critique théâtral Jules Claretie (56).

L'intérêt de Fréchette pour l'art de la scène remonte à ses années d'études à l'Université Laval et à l'écriture de sa pièce Félix Poutré (1862). Fréchette continuera à écrire pour la scène, y compris lors de son séjour à Chicago. Parmi ses pièces, il faut noter «Les fiancés de l'Outaouais », «Tête à l'envers» et sa pièce restée inachevée «La Confédération ». Malheureusement, ces trois pièces, jouées sur scène à Chicago, sont détruites lors d'un incendie (Bélanger). Après son retour au Québec, Fréchette écrit deux pièces : Papineau et Le retour de l'exilé (1880). Il écrit aussi de petites comédies en un acte, demeurées inédites, comme « Notables de village », jouée à la salle de Jacques Cartier le 19 janvier 1863, « Une journée à l'Hôtel de Canada », montée à l'Académie de musique en décembre 1881, et "Change pour change », interprétée par les étudiants du collège de Nicolet en 1886 (voir Bélanger). La dernière pièce de Fréchette, Véronica, est lue au Monument-National en 1898 et jouée à Montréal en 1903. Parmi ces pièces, les « drames historiques » se démarquent clairement. 


\section{L’adaptation théâtrale : Félix-Poutré (1862)}

Tout comme en France ${ }^{7}$, l'adaptation théâtrale est une pratique courante au Québec au dixneuvième siècle. Plusieurs pièces sont inspirées ou tirées de romans historiques à succès telles que Les anciens Canadiens de Philippe Aubert de Gaspé, L'intendant bigot de Joseph Marmette et Rodolphe Tanguay, créée en 1873, ainsi qu'Une de perdue, deux de trouvées de Georges Boucher de Boucherville. En absence des pièces originales ${ }^{8}$, ces adaptations théâtrales peuplent les salles et permettent aux jeunes auteurs d'apprendre à écrire pour la scène, à connaître les enjeux de l'écriture théâtrale et à entamer leur carrière, comme c'est le cas pour Fréchette dont la première pièce, Félix Poutré, est une adaptation théâtrale. Fréchette adapte le texte autobiographique de Félix-Poutré intitulé Échappé de la potence : souvenirs d'un prisonnier d'État canadien en 1838 (1862) qui raconte l'histoire d'un jeune homme en prison durant l'insurrection de 1838 mais qui échappe à la potence en feignant la folie. Fréchette en fait un «drame historique » en trois actes en choisissant le titre Félix Poutré ou l'Échappé de la potence; épisode de la révolution canadienne de 1838. Lors de sa publication en 1871, ce titre est raccourci par son éditeur Beauchemin, qui réduit aussi la pièce à quatre actes (voir Bélanger).

Le 22 novembre 1862, la pièce, montée par «Le Théâtre des familles », troupe « d'amateurs distingués » (voir Robert), est présentée pour la première fois à la Salle de musique de Québec, appelée aussi à l'époque la Salle de la rue Saint-Louis. « Construite en 1853 et dessinée par l'architecte Charles Baillargé », elle compte mille cinq cents places. Dès le 29 septembre 1862, la presse écrite (Le Canadien et Le journal de Québec) se montrent attentifs à la production ${ }^{9}$. Le 30 septembre 1862, Le journal de Québec annonce : « M.L.H. Fréchette vient de terminer, pour le théâtre de cette ville, un drame historique en trois actes, avec prologue qu'il a intitulé 'Félix Poutré, ou l'échappé de la potence; épisode de la révolution canadienne de 1838" » (2). Le journal ajoute que la pièce sera jouée par des acteurs amateurs vers la mi-novembre et que les comédiens seront bientôt choisis, sauf le typographe M. Paul Dumas qui jouera dans le rôle-titre. Bien que les journaux annoncent d'abord le jeudi 27 novembre 1862, cette date est avancée au samedi 22 (Le Canadien, 17 novembre 1862,2), ce qui ne donne qu'à peu près un mois et demi de répétition avant le spectacle.

Le journal de Québec décrit la pièce comme un «grand drame historique » en trois actes et un prologue, « tiré des Mémoires d'un prisonnier d'état canadien ». Dans le reste de l'annonce, on trouve le titre des actes et le prix d'entrée allant de cinquante cents (les galeries et parterre) à 
soixante-quinze cents (les loges d'orchestre). Malgré le peu de temps pour la préparation, les journaux confirment le succès de la soirée, ainsi dans Le journal de Québec du 25 novembre 1862. Le Morning Chronicle and Commercial and Shipping Gazette fait paraitre un compte rendu :

"Félix Poutré" a three-act drama, by Mr. L. H. Fréchette, of this city, was performed at the Music Hall, on Saturday night, before a crowded house. As a dramatic production, it was very successful. It was creditable to the young and talented author as a literary effort. The chief role was sustained with great power and effect by an amateur of considerable merit, Mr. Paul Dumas. The minor characters were well played, for amateurs ; and the stage accessories were admirable. (24 nov. 1862, 2)

La pièce sera montée à nouveau dans la Salle de Jacques Cartier le 19 janvier 1863 avec une nouvelle petite comédie en un acte de Fréchette intitulée « Notables de village ».

Fréchette s'inscrit pleinement dans le contexte du patriotisme québecois au dix-neuvième siècle, « premier auteur dramatique à mettre en scène les Rébellions » (Robert 18). Un grand nombre de poèmes et de romans canadiens-français expriment l'amour de la patrie durant cette époque. Les romans historiques mettant en scène le portrait glorieux de la Nouvelle-France ou les troubles de 1837 prennent leur essor. Tel que le souligne Daniel Mativat dans Le métier d'écrivain au Québec (1840-1900). Pionniers, nègres ou épiciers des lettres ?, écrire est perçu comme un acte patriotique et tout ce qui sert cette cause est appelé littérature : « L'écrivain n'est plus un libre créateur, c'est un agent de transformation sociale qui réactualise l'Histoire et les traditions populaires pour assurer l'avenir de la race et de la langue » (284).

\section{Papineau et Le retour de l'exilé}

En 1880, Fréchette, s’inspirant encore des évènements de 1837 et 1838, revient au théâtre avec deux pièces : Papineau et Le retour de l'exilé. Papineau, drame historique canadien en quatre actes et neuf tableaux, dont le titre porte le nom de son personnage principal, commence avec cette dédicace : « À mon vieil ami, M. Philippe Pacaud, le seul survivant des personnages historiques de cette pièce ». La pièce met en scène les batailles de Saint-Denis et de Saint-Charles (novembre 1837), qui ont fini par la fuite de plusieurs «patriotes » aux États-Unis, y compris Louis-Joseph Papineau et Wolfred Nelson, leurs chefs. Elle débute à Saint-Denis, dans l'appartement de George Laurier (sans «s») en octobre 1837, juste avant l'insurrection des Patriotes. James Hastings, officier anglais, appelé autrefois Jimmy, revient d'Europe (Angleterre et France) après une absence de cinq ans et retrouve son ancien compagnon de collège George. 
James rencontre ensuite Rose, sœur de George, pour qui il éprouve des sentiments amoureux. Jusqu'ici, la pièce rappelle le roman d'Aubert de Gaspé, Les anciens Canadiens (1863), mais le développement et la fin de la pièce sont différents. Rose, contrairement à Blanche dans le roman, accepte la demande en mariage de James et finit par épouser «l'ennemi » à la fin de la pièce. Plusieurs scènes des assemblés des Patriotes chez le docteur Nelson figurent dans la pièce où le personnage Papineau joue un rôle important. Bien qu'il soit le chef des Patriotes et qu'on souligne à plusieurs reprises son côté héroïque, Papineau est décrit comme « un homme d'État pacifique » et comme un homme de raison. Ses caractéristiques humaines sont soulignées à plusieurs reprises : il est fiable, généreux, inquiet des troubles et, surtout, religieux. Notons que Papineau n'est pas la seule figure historique qui occupe les drames historiques canadiens-français de la fin du dixneuvième siècle. Louis Riel occupe également le centre de plusieurs pièces de théâtre, par exemple dans Riel, tragédie en quatre actes (1886) d'Elzéar Paquin, et Riel (1886) de Charles Bayer et Ernest Parage.

Dans le Retour de l'exilé, drame en cinq actes et huit tableaux, le dramaturge adapte à la scène le roman La bastide rouge (1860) d'Élie Berthet, écrivain français, lui donne une forme dialoguée, situe l'action au Canada français, notamment à Sillery près de Québec, et change le nom des personnages. La pièce porte sur Auguste DesRivières. Pendant vingt-deux ans d'expulsion, Auguste a parcouru plusieurs pays (la pièce le présente comme un voyageur) et rentre au pays pour réclamer l'héritage qu'il avait confié à Jolin, ancien commis de son père. Ce dernier, méchant de la pièce et chef des brigands, profite de la fortune et n'a pas l'intention de restituer les biens en question. Contrairement à Félix-Poutré et à Papineau, l'intrigue se déroule après les Rébellions. Le mélange des registres et des genres attire l'attention. La pièce donne plus l'impression d'un mélodrame que d'un drame historique en raison de ses personnages et des actions malheureuses et inattendues qui s'enchaînent. L'action se déroule majoritairement la nuit et le ton mystérieux domine dès le début. Dans une scène de tentative de meurtre, Jolin, par crainte de perdre les biens qui lui ont été confiés, ordonne à un brigand de tuer Auguste durant son sommeil. Un certain Adrien avertit la veuve Mme Saint Valier et sa fille Blanche qui vivent sous le toit de Jolin. Ce dernier planifie d'épouser Blanche contre sa volonté alors qu'elle est amoureuse d'Adrien. On apprend, par la suite, qu'Adrien est le fils d'Auguste. La pièce se termine par une fin heureuse grâce à une lettre qui atteste son héritage. Jolin est arrêté par la police, Auguste récupère sa fortune et épouse Blanche. 
Papineau et Le retour de l'exilé remplissent les salles à Montréal pendant six soirées et une matinée. Si l'on en croit les journaux de l'époque, en particulier Le courrier de Montréal, la création des pièces entre le 7 et le 12 juin 1880 fut un évènement théâtral et marqua le développement du théâtre francophone. Les articles de journaux s'empressent de remarquer que, pour la première fois, deux pièces du même auteur canadien sont jouées pendant sept représentations consécutives (Larrue, «Les créations »). Pour donner vie à ses pièces, Fréchette apporte un soin jaloux à la distribution des rôles et aux décors ${ }^{10}$. Il confie le rôle principal à la célèbre cantatrice belge Jehin-Prume et le rôle de Papineau à Paul Dumas, qui avait auparavant incarné Félix Poutré (voir l'annonce de la pièce dans Le canard, 5 juin 1880, 4). Pour le décor, le dramaturge travaille avec l'artiste peintre montréalais René Garand. D'après le compte rendu du Peuple, journal hebdomadaire montréalais, intitulé «Deux Premières», le succès de ces représentations est le fruit du talent des comédiens, des techniciens de théâtre et de dramaturge Fréchette (12 juin 1880,5).

\section{Louis Fréchette et Sarah Bernhardt}

L'apport de Fréchette au développement du théâtre canadien-français marque également les relations d'amitiés qu'il a établies avec des artistes étrangers, lorsque Montréal est devenu une métropole culturelle visitée par de nombreux comédiens ${ }^{11}$. Lorsque la célèbre actrice française Sarah Bernhardt vient à Montréal pour la première fois en décembre 1880, Fréchette est parmi les gens qui l'accueillent. Ceci marque le début d'une amitié entre l'écrivain et l'artiste ${ }^{12}$.

Bernhardt a continuellement occupé l'actualité des journaux canadiens et étatsuniens de l'époque. La presse annonce la première rencontre de Bernhardt avec les Montréalais des mois à l'avance. Le 8 juillet 1880 dans L'opinion publique, Anthony Ralph décrit le répertoire de sa troupe comme étant «brillant et complet, [...] le nec plus ultra de ce que la langue française a produit de plus pur, de plus harmonieux » et souligne le talent exceptionnel de Bernhardt : sa façon d'utiliser sa voix, ses gestes, ses mimiques et sa capacité d'entrer dans la peau des personnages (Phèdre, Marie Stuart, Andromaque, Desdémona, la dame aux Camélias). L'article conclut ainsi : «O Montréalais! Lorsque vous la verrez, vous ferez comme moi, vous l'aimerez ( 8 juillet 1880, $3){ }^{13}$

Malgré les mandements de l'évêque ${ }^{14}$ et la condamnation des productions de la compagnie de Bernhardt qui paraissent dans la presse conservatrice, le théâtre fait salle comble pendant trois 
soirées et une matinée. On annonce la visite de Bernhardt et les créations d'Adrienne Levouvreur, Frou-frou, La dame aux Camélias et Hernani comme " un succès bœuf ${ }^{15}$. Après cette visite, Fréchette entretiendra son amitié avec l'actrice par correspondance, puis lors des visites de Bernhardt à Montréal en 1891 et en 1896 (voir Béraud). Fréchette compose un poème pour la vedette et a même l'idée d'écrire une pièce de théâtre pour l'actrice lors de cette première rencontre. Il mènera ce projet à terme une vingtaine d'années plus tard sous la forme d'un mélodrame romantique en cinq actes, Véronica. La pièce sera produite sur une scène montréalaise en 1903. Elle n'a jamais été interprétée par Bernhardt, bien que son nom apparaisse dans la publicité : «Véronica, écrite à la demande de Sarah Bernhardt» (La presse, Les débats, Le canard, janvier 1903).

\section{Conclusion}

Bien qu'il soit aujourd'hui tombé dans l'oubli, le théâtre de Fréchette a transformé le théâtre d'expression française du dernier quart de dix-neuvième siècle au Québec. D'après Robert, Le retour de l'exilé « a servi de matrice à une série d'autres mélodrames à succès » (25-26). Robert ajoute également une série de pièces et adaptations portant sur le thème des Rébellions apparues

après les pièces de Fréchette, notamment au début du vingtième siècle. Fréchette a donc non seulement influencé ses contemporains, mais aussi ses successeurs.

\section{Ouvrages cités}

Bélanger, Reine. «Félix-Poutré, drame de Louis Fréchette». Dictionnaire des æuvres littéraires du Québec. Montréal : Fides, 1980.

Béraud, Jean. 350 ans de théâtre au Canada français. Montréal : Cercle du livre de France, 1958. 7-106.

Bisson, Margaret Mary. «Le théâtre français à Montréal, 1878-1931 ». Mémoire de maîtrise, Montréal : Université McGill, 1931.

Entretien avec Lucie Robert. Société des dix. https://fb.watch/3uJboxUDC1/.Consulté le 5 janvier 2021.

Fréchette, Louis. Félix-Poutré. Montréal : Leméac, 1974.

---. Le retour de l'exilé. Montréal : Leméac, 1974.

---. Papineau. Montréal : Leméac, 1974. 
---. Véronica. Montréal : Leméac, 1974.

Germain, Jean-Claude. La double vie littéraire de Louis Fréchette; suivi de, Une brève histoire du conte au Québec. Montréal : Hurtubise, 2014.

Laflamme, Jean et Rémi Tourangeau. Église et le théâtre au Québec. Montréal : Fides, 1979.

Larrue, Jean-Marc. «Les créations scéniques de Louis-Honoré Fréchette : juin 1880 ». Theatre Research in Canadal Recherches théâtrales au Canada 7.2 https://journals.lib.unb.ca/index.php/TRIC/article/view/7391. Consulté le 5 janvier 2021.

---. Le théâtre à Montréal à la fin du XIXe siècle. Montréal : Fides, 1981.

Léger, Danielle. «L'histoire du théâtre à Montréal racontée sur le web par BAnQ et trois universités québécoises ». Documentations et bibliothèques 65.1 (2019) : 37-45.

Lemire, Maurice et Denis Saint-Jacques, dirs. La vie littéraire au Québec, Tome IV (1870-1894). Québec : PUL, 1999. 351-368.

Mativat, Daniel. Le métier d'écrivain au Québec (1840-1900). Pionniers, nègres ou épiciers des lettres? Montréal : Triptyque, 1996.

Poutré, Félix. Échappé de la potence: souvenirs d'un prisonnier d'État canadien en 1838. Montréal : De Montigny, 1862.

Robert, Lucie. « L’art de transformer une défaite en victoire. La représentation des Rébellions dans le théâtre de Louis Fréchette ». Bulletin d'histoire politique 12.1 (2003) :16-27.

Tremblay, Alex. «L'enracinement du théâtre et de la musique à Québec au XIX ${ }^{\mathrm{e}}$ siècle ». Histoire Québec 20.3 (2015) : 10-16.

Viveiros, Geneviève de. "'Les romans mis en pièces" : étude sur la pratique de l'adaptation théâtrale à la fin du XIXe siècle. Les cas d'Émile Zola (1873-1902) ». Thèse de doctorat, Université de Toronto, 2009.

\footnotetext{
Notes

${ }^{1}$ Ma recherche porte sur les périodiques numérisés sur la banque de données Numérique.banq.qc.ca et Canadiana.org.

${ }^{2}$ Le théâtre de Marchand donne lieu à un projet doctoral poursuivi par Umut Incesu à l'Université Western Ontario, sous la direction de Geneviève de Viveiros.

${ }^{3}$ En 2019, Lucie Robert mentionne la méprise envers l'histoire du théâtre québécois (Entretien, Société des dix).

${ }^{4}$ Le Laboratoire d'histoire et de patrimoine de Montréal (LHPM) de l'UQAM met en valeur les documents d'archives théâtrales de BAnQ, jusqu'à présent quasiment inexplorés. Voir l'article de Danielle Léger.

5 Plusieurs périodiques annoncent cette nouvelle, entre autres l'article d'Ernest Tremblay sous le titre «Un événement », publié dans Le peuple, 12 juin 1880, p. 4.

${ }^{6}$ Voir les documents d'archives de la Bibliothèque et Archives nationales du Québec, où l'on trouve des lettres entre Pamphile Le May (1837-1918), Benjamin Sulte (1841-1923), Joséphine Marchand-Dandurand (1861-1925) et même
} 
une lettre envoyée au dramaturge Émile Augier (1820-1899) datant du 4 juin 1877 (BAnQ Québec, Fonds LouisHonoré Fréchette, Cote : MSS231, P133).

${ }^{7}$ L'adaptation des romans en pièce de théâtre se répandait en Europe et surtout en France. Voir Geneviève de Viveiros. ${ }^{8}$ Selon Jean-Marc Larrue (Le théâtre), c'est surtout à partir de 1860 que l'écriture dramatique connaît un essor au Québec grâce au nombre des spectateurs qui s'élargit. Larrue note l'écriture d'une quarantaine de pièces entre 1870 et 1890 .

${ }^{9}$ On lit dans Le Canadien: «On nous prie de dire que la pièce inspirée à M. L. H. Fréchette par l'épisode de la révolution canadienne dont M. Félix Poutré est le héros sera prête pour la représentation vers la mi-novembre », 29 septembre 1862, p. 2.

10 Jean-Marc Larrue explique le côté novateur de ces productions de la manière suivante : «Jamais auparavant personne n'avait accordé autant de soins, d'énergie et de moyens à la préparation de créations locales. Et jamais la presse ne s'était montrée aussi attentive à des spectacles amateurs ». Le Nouveau Monde, 15 juin 1880, p. 2. cité dans l'article de Jean-Marc Larrue (1986).

${ }^{11}$ Mentionnons, entre autres, les multiples visites des comédiennes françaises Gabrielle-Charlotte Réju, dite Réjane (1856-1920) et Sarah Bernhardt (1844-1923). Ces actrices jouent plusieurs pièces du répertoire français. Il y a aussi la visite des troupes de théâtre louisianaises, par exemple La Troupe St-Louis de la Nouvelle Orléans, alors que plusieurs troupes françaises s'établissent au Québec comme les troupes de Maugard, de Frantz Jehin Prume et des Génot. Pour en savoir davantage, consulter Margaret Mary Bisson.

${ }^{12}$ Dans les documents d'archives de la Bibliothèque et Archives nationales du Québec, se trouvent des lettres et télégrammes envoyés de Paris où Bernhardt remercie Fréchette de son accueil lors de ses visites (BAnQ Québec, Fonds Louis-Honoré Fréchette, Cote : MSS231, P133).

${ }^{13}$ Anthony Ralph consacre plusieurs articles à la tournée de l'actrice dans L'opinion publique. Voir, entre autres, le 28 octobre 1880, p. 2 et le 11 novembre 1880, p. 3.

${ }^{14} \mathrm{Vu}$ comme «dangereux » par l'Église, car il met en péril les valeurs traditionnelles du mariage et de la famille, le théâtre subit constamment les attaques du clergé tout au long du siècle. Voir Jean Laflamme et Rémi Tourangeau.

${ }^{15}$ Anonyme, «Sara Bernhardt à Montréal », Le canard, 31 décembre 1880, p. 4 (L'article emploie le nom « Sara » pour l'actrice). 